\title{
Does MDR1 Analysis Predict Medical Therapy Modifications in Patients with Atrial Fibrillation?
}

\author{
Ayhan Akoz1, Kenan Ahmet Turkdogan1, Bekir Dagli1, Mucahit Kapci1, Mucahit Avcil1, Gokay Bozkurt² and Ali Duman1
}

\begin{abstract}
Objective: To evaluate the effects of multi-drug resistance gene (MDR1) gene factor which is significant in medicinereceptor relationship, on readmission to the emergency department (ED) and medical therapy modifications in patients with atrial fibrillation (AF) readmitting to the emergency department.

Study Design: Descriptive, analytical study.

Place and Duration of Study: Department of Emergency Medicine, Adnan Menderes University, Aydin, Turkey, from January 2016 to January 2017.

Methodology: Fifty patients who did not have AF with rapid ventricular response, and 32 controls have been included in the study. Electronic recording system of the hospital was checked regularly to detect any readmission of these patients due to palpitation; and they were asked whether they had any ED readmission and any changes in medical therapy by calling them during the one-year period. Then, MDR1 1236TC, 2677TG and 3435TC gene analyses and medical treatment regimens of the patients after 1 year were compared.

Results: No significant differences were found neither between the study and the control group nor between the genders in the study group regarding the results of MDR1 gene analyses. Besides, there were no differences in medical treatment regimens compared to MDR1 gene analyses in the group with AF. There were no statistically significant differences in the results of MDR1 gene analysis in patients whose medical treatment regimen had been changed during the one-year period.

Conclusion: MDR1 gene analyses did not have any significant effect on the development of AF, readmission to the ED and modification of the treatment regimenin the Turkish population.
\end{abstract}

Key Words: Multi-drug resistance gene, Atrial fibrillation, Drug resistance, Emergency department, Genetic mutation.

\section{INTRODUCTION}

Atrial fibrillation $(\mathrm{AF})$ is an irregular and often rapid heart rate. Despite good progress in the management of patients with $A F$, this arrhythmia remains one of the major causes of sudden death, heart failure, stroke and cardiovascular morbidity in the world. Furthermore, the number of patients with $A F$ is predicted to rise steeply in the coming years. ${ }^{1}$ Estimates suggest, AF prevalence is of approximately $3 \%$ in adults aged 20 years or older, with greater prevalence in older persons and in patients with conditions such as heart failure, valvular heart disease, hypertension, coronary artery disease (CAD), chronic kidney disease (CKD) or diabetes mellitus. ${ }^{2-4}$ The increase in AF prevalence can be attributed both to better detection of silent $A F$, alongside increasing age and conditions predisposing to AF.5,6 Several AF risk variants are also associated with cardio-embolic or ischemic stroke, possibly due to silent $A F^{7,8}$ Changes in atrial action potential characteristics, ${ }^{9}$ atrial remodeling and modified penetration of rare gene defects ${ }^{10}$ have

Department of Emergency Medicinel / Medical Genetic ${ }^{2}$,

Adnan Menderes University, Aydin, Turkey

Correspondence: Dr. Ayhan Akoz, Department of Emergency

Medicine, Adnan Menderes University, Aydin, Turkey

E-mail:akozayhan@gmail.com

Received: June 23, 2018; Revised: February 04, 2019;

Accepted: March 18, 2019 been suggested as potential mechanisms mediating increased AF risk in carriers of common gene variants. ${ }^{11-13}$

P-glycoprotein which is a product of multi-drug resistance gene (MDR1) is an important protein of the cell membrane that pumps xenobiotics (substances not a natural constituent of the organism) out of cells through an ATP-dependent mechanism. P-glycoprotein has some role in the removal of various medications since it has a wide range of substrate specificity. ${ }^{14}$ There are several studies and evidences suggesting P-glycoprotein is an important pharmacokinetic factor for cardiac glycosides, especially digoxin. Studies on animals and humans showed that P-glycoprotein is effective on the absorption and tissue distribution of cardiac glycosides. It was shown that $50 \%$ of variation of bio-availability of digoxin after oral administration in healthy volunteers is associated with the intestinal expression of P-glycoprotein. ${ }^{15}$

It was aimed in this study to evaluate the effects of MDR1 gene factor, which is significant in medicinereceptor relationship, on readmission to the emergency department and medical therapy modifications in patients with AF readmitting to the emergency department.

\section{METHODOLOGY}

This descriptive study was conducted at the Department of Emergency Medicine, Adnan Menderes University, Aydin, Turkey, from January 2016 to January 2017. In 
this study, 32 patients who did not have AF were included as the control group from January 2016 to January 2017 . Fifty patients with AF who admitted to the emergency department with no rapid ventricular response included as the study group. Electronic recording system of the hospital was checked regularly to detect any readmission of these patients due to palpitation. Moreover, they were asked whether they had any emergency department readmission and any changes in medical therapy by calling them during the one-year period. Then, MDR1 1236TC, 2677TG and $3435 \mathrm{TC}$ gene analyses and medical treatment regimens of the patients after one year were compared.

Exclusion criteria were heart rate under 120 bpm, hemodynamically unstable patients, atrio-ventricular-block (second or third degree), ventricular rhythm disorder, acute coronary syndrome, kidney failure, valvular heart disease, malignancy, refusal to participate in the study, and death during the follow-up period.

The subject was approved by the Medical Ethics Committee of Adnan Menderes University, and all patients have signed an informed consent.

A commercially available isolation kit (Roche-High Pure PCR TemplatePreparation Kit) was used to isolate DNA from peripheral blood samples. Two hundred $\mu \mathrm{l}$ of blood sample was incubated at $70^{\circ} \mathrm{C}$ after vortexing it with $200 \mu \mathrm{l}$ of binding buffer and $40 \mu \mathrm{l}$ of proteinase $\mathrm{K}$, then with 100 $\mu \mathrm{l}$ of isopropanol and $200 \mu \mathrm{l}$ of elution buffer. Five hundred $\mu \mathrm{l}$ of inhibitor was centrifuged with removalbuffer at $8,000 \mathrm{~g}$ and with wash buffer at $13,000 \mathrm{~g}$ for 1 min in the filter tube provided in the kit. DNA isolation was achieved with centrifugation with elution buffer at $8,000 \mathrm{~g}$ for $1 \mathrm{~min}$ after $10 \mathrm{mins}$ of incubation at $70^{\circ} \mathrm{C}$.

SNP test was performed using 5 to $50 \mathrm{ng}$ DNA for each reaction by paying attention to $A_{260} / A_{280}$ absorbance rate to be between 1.8 and 2.0 for the purity of DNA isolated.

For MDR1 gene SNPs (rs1128503 1236 T>C, rs2032582 $2677 \mathrm{~T}>\mathrm{G} / \mathrm{A}$ ve rs1045642 $3435 \mathrm{~T}>\mathrm{C}$ ), spinning was applied by adding $105 \mu$ l water to lyophilised form. Then, the reaction mixture for Real-Time PCR process for an MDR1 SNP was prepared.

Fifteen $\mu \mathrm{l}$ of the mixture prepared for Real-Time PCR reaction to detect MDR1 alleles was spread on the plate, and $5 \mu \mathrm{l}$ DNA was added so final volume of $20 \mu \mathrm{l}$ was reached. These plates were loaded to Roche LC480 device and analysed following the appropriate protocol. "Melting Curve Genotyping" program for Light Cycler 480 was used for data analysis (Figures 1-3).

Descriptive statistics for categorical variables were stated as number $(\mathrm{n})$ and percentage (\%). The Chi-square test was used to compare data between groups. The t-test was used for group comparisons of quantitative which were expressed as mean $\pm S D$. A value of $p<0.05$ was accepted as statistically significant (2-tailed test).

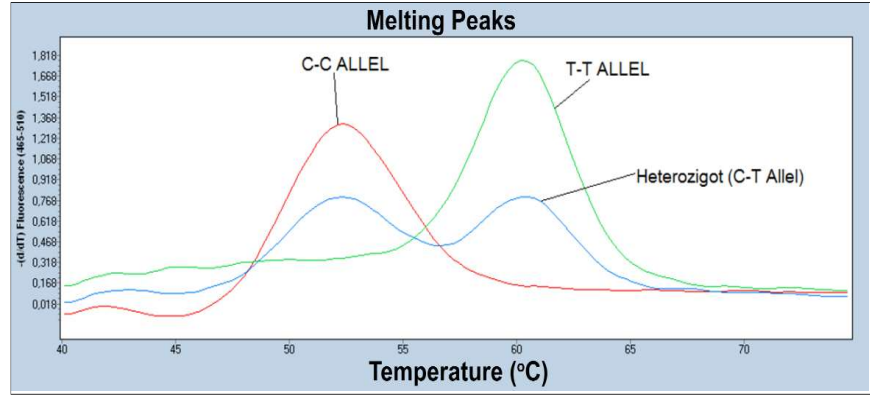

Figure 1: For MDR1 rs1128503 $1236 \mathrm{~T}>\mathrm{C}$.

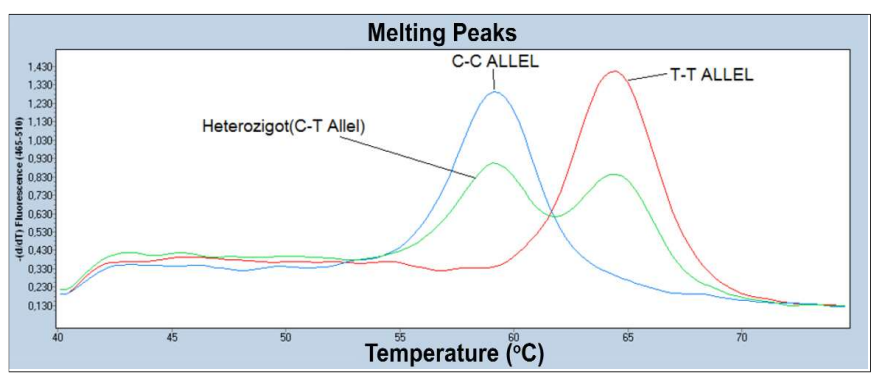

Figure 2: MDR1 rs2032582 2677T>G/A

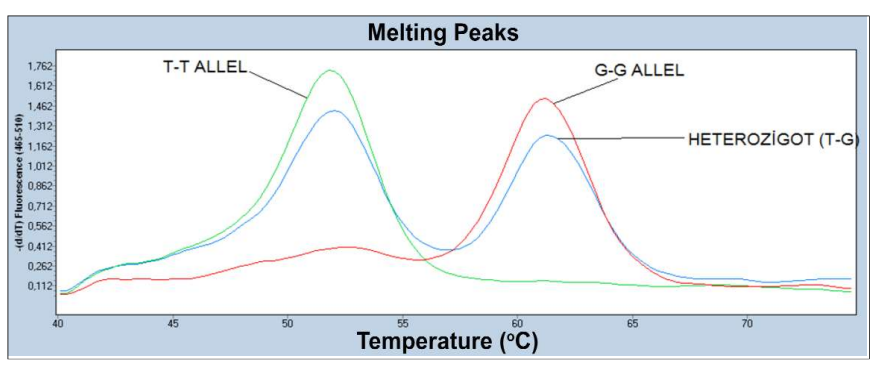

Figure 3: MDR1 rs2032582 2677T>G/A.

\section{RESULTS}

Eleven (34.4\%) patients in the control group and 17 $(34.0 \%)$ patients in the study group were females. Mean age of the patients included in the control and study groups were $63 \pm 22.3(n=32)$ years and $70.14 \pm 14.4$ $(n=50)$ years, respectively; the difference was statistically not significant $(p=0.151)$.

Comparison of the study and the control group related to gene analyses are given in Table I-III. The groups were found to have no statistically significant difference with respect to MDR1 1236TC gene $(p=0.313$, Table I). The groups were found to have no statistically significant difference with respect to MDR1 2677TG gene $(p=0.167$, Table II). The groups were found to have no statistically significant difference with respect to MDR1 $3435 T C$ gene $(p=0.716$, Table III). The groups were found not to differ with regard to 1236TC, 2677TG and $3435 \mathrm{TC}$ in both male ( $p=0.739 ; 0.834 ; 0.685$, respectively) and female patients ( $p=0.853 ; 0.692 ; 0.489$, respectively).

There were no significant differences in the distribution of 1236TC, 2677TG and 3435TC gene analyses regarding 
Table I: Comparison of the study and the control group related to gene analyses of MDR1 1236TC gene.

\begin{tabular}{l|c|c|c|c}
\hline \multirow{2}{*}{ Groups $(n=82)$} & \multicolumn{3}{|c|}{ MDR1 1236TC } & \multirow{2}{*}{ p-value } \\
\cline { 2 - 4 } & CC Allele & Heterozygote & TT Allele & \\
\hline Control $(n=32)$ & $7(21.9 \%)$ & $15(46.9 \%)$ & $10(31.3 \%)$ & 0.313 \\
AF $(n=50)$ & $18(36.9 \%)$ & $22(44.0 \%)$ & $10(20.0 \%)$ & \\
\hline
\end{tabular}

Table II: Comparison of the study and the control group related to gene analyses of MDR1 MDR1 2677TG gene.

\begin{tabular}{l|c|c|c|c|c}
\hline \multirow{2}{*}{ Groups $(n=82)$} & \multicolumn{4}{|c|}{ MDR1 2677TG } & \multirow{2}{*}{ p-value } \\
\cline { 2 - 5 } & AA Allele & GG Allele & Heterozygote & TT Allele & \\
\hline Control $(n=32)$ & $1(3.1 \%)$ & $6(18.8 \%)$ & $17(53.1 \%)$ & $8(25.0 \%)$ & 0.167 \\
AF $(n=50)$ & $2(4.0 \%)$ & $21(42.0 \%)$ & $19(38.0 \%)$ & $8(16.0 \%)$ & \\
\hline
\end{tabular}

Table III: Comparison of the study and the control group related to gene analyses of MDR1 MDR1 MDR1 3435TC gene.

\begin{tabular}{l|c|c|c|c}
\hline \multirow{2}{*}{ Groups $(n=82)$} & \multicolumn{3}{|c|}{ MDR1 3435TC } & \multirow{2}{*}{ p-value } \\
\cline { 2 - 4 } & CC Allele & Heterozygote & TT Allele & \\
\hline Control $(n=32)$ & $13(40.6 \%)$ & $14(43.8 \%)$ & $5(15.6 \%)$ & \multirow{2}{*}{0.716} \\
AF $(n=50)$ & $20(40.0 \%)$ & $25(50.0 \%)$ & $5(10.0 \%)$ & \\
\hline
\end{tabular}

Table IV: Comparison of the study group according to the treatment modification in gene analyses of MDR1 1236TC gene.

\begin{tabular}{l|c|c|c|c}
\hline \multirow{2}{*}{ AF $(n=50)$} & \multicolumn{3}{|c|}{ MDR1 1236TC } & p-value \\
\cline { 2 - 4 } & CC Allele & Heterozygote & TT Allele & \\
\hline $\begin{array}{l}\text { Patients with no } \\
\text { treatment modification } \\
(\mathrm{n}=32)\end{array}$ & $12(37.5 \%)$ & $14(43.8 \%)$ & $6(18.8 \%)$ & 0.939 \\
$\begin{array}{l}\text { Patients with treatment } \\
\text { regimen modification } \\
\text { (Digoxin added })(\mathrm{n}=18)\end{array}$ & $6(33.3 \%)$ & $8(44.4 \%)$ & $4(22.2 \%)$ & \\
\hline
\end{tabular}

beta blocker, calcium channel blocker and digoxin use in patients with atrial fibrillation $(p=0.141 ; 0.160 ; 0.879$, respectively). Female patients with atrial fibrillation were found to have no significant differences in the distribution of 1236TC, 2677TG and 3435TC gene analyses with respect to the medical treatment $(p=0.415 ; 0.753 ; 0.302$, respectively). Male patients with atrial fibrillation were also found to have no significant differences in the distribution of 1236TC, 2677TG and 3435TC gene analyses with respect to the medical treatment ( $p=0.202 ; 0.115 ; 0.353$, respectively).

None of the patients in the control group had AF or given medical treatment during the 1-year study period. However, $18(36.0 \%)$ patients in the study group had emergency department readmissions and needed treatment regimen modifications or multi-drug use. MDR1 gene analyses of these patients showed no statistically significant difference in 1236TC (Table IV), 2677 TG and 3435TC ( $p=0.939 ; 0.715 ; 0.411$, respectively).

\section{DISCUSSION}

This study is the first one evaluating the effect of MDR1 gene mutation on the requirement of digoxin therapy in the Turkish population. It was shown that $50 \%$ of variation of bio-availability of digoxin after oral administration in healthy volunteers is associated with the intestinal expression of P-glycoprotein. Distribution and elimination of digoxin is highly affected in guinea pigs with MDR1a deficiency. It should be noted that the difference in the distribution and elimination of digoxin between MDR1a $(-/-)$ and MDR $1 \mathrm{a}(+/+)$ animals is 1.9 times. It was also shown that MDR1a deficient guinea pigs have 35 times higher concentrations of the drug in the brain tissue. 15

Hence, one can think that differences in P-glycoprotein gene expression in transfer of P-glycoprotein substrates should be evaluated independently for any single tissue. Besides, concentration of the drug can be a determining factor because P-glycoprotein-related transfer can reach saturation. The transfer is supposed to be maximal when higher concentrations were given through the gastrointestinal lumen, and limiting contribution of P-glycoprotein absorption will be less evident. Additionally, serum concentrations of the drug will be 2 to 3 times lower than the intestinal concentration when the drug reached systemic circulation. Thus, the effect of P-glycoprotein gene expression in the transfer of P-glycoprotein substrates in the blood will be higher compared to moderate effect observed in the intestinal tract. Grenier suggested that $50 \%$ variance in digoxin absorption related to intestinal P-glycoprotein expression is a result of MDR1 polymorphism. Grenier reported that intestinal expression of exon 26 C3435 T genotype is 2 times higher. 16 Hoffmeyer reported that, plasma digoxin concentration is more consistent in TT group unlike CC gentotypein C3435T group.17 So, concentrations of digoxin is supposed to be higher when it is used through the mouth in Caucasian patients with 3435TT genotype (TT>TC>CC). Nineteen (59.4\%) of the 32 patients included in the control group in the current study had polymorphisms affecting digoxin absorption (MDR1 3435TC, MDR1 3435TT). MDR1 3435TC and MDR1 $3435 \mathrm{TT}$ were found in $14(43.8 \%)$ patients and 5 $(15.6 \%)$ patients in the control group, respectively. Briefly, the results of the present study support those of Grenier's study because the occurrence rate of polymorphisms affecting the variance of intestinal absorption of digoxin was found $59.4 \%$ similar to that found in Grenier's study (50\%).

Subjects with TT genotype in 3435 locus given digoxin orally have higher and more stable digoxin concentrations than those with CC genotype do. Kurata et al. also reported similar results. They have found that definitive bioavailability of digoxin in subjects with 2677TT/3435TT (having homozygote thymine in both 2677 and 3435 loci) genotype was significantly higher than that measured in those with 2677GG / 3435CC genotype. ${ }^{18}$ It was found that maximum bioavailability had the highest level in those with homozygote mutant alleles (mean: 81.7\%), moderate in those with heterozygote mutant alleles and the lowest in those with homozygote normal alleles (67.6\%). Besides, they have found renal clearance of digoxin was approximately $32 \%$ 
lower in the subjects with 2677TT/3435TT genotype than in those with 2677GG / 3435CC genotype, and the subjects with 2677GT/3435CT genotype had a level between the two. These results indicate that impairments in the intestinal expression and renal secretion of digoxin in the subjects with single nucleotide polymorphisms occurs simultaneously. 19

\section{CONCLUSION}

MDR1 1236TC, 2677TG and 3435TC gene analyses do not have any significant effect on the development of AF and readmission to the emergency department and modification of the treatment regimen in those with $\mathrm{AF}$; some other factors may have more influence in the Turkish population.

\section{REFERENCES}

1. Kirchhof P, Benussi S, Kotecha D, Ahlsson A, Atar D, Casadei B, et al. 2016 ESC Guidelines for the management of atrial fibrillation developed in collaboration with EACTS. Eur $J$ Cardiothorac Surg 2016; 50:e1-e88

2. Mc Manus DD, Rienstra M, Benjamin EJ. An update on the prognosis of patients with atrial fibrillation. Circulation 2012; 126:e143-6.

3. Ball J, Carrington MJ, McMurray JJ, Stewart S. Atrial fibrillation: Profile and burden of an evolving epidemic in the 21st century. Int J Cardiol 2013; 167:1807-24

4. Oldgren J, Healey JS, Ezekowitz M, Commerford P, Avezum A, Pais $P$, et al. Variations in cause and management of atrial fibrillation in a prospective registry of 15,400 emergency department patients in 46 countries: The RE-LY Atrial Fibrillation Registry. Circulation 2014; 129:1568-76.

5. Kishore A, Vail A, Majid A, Dawson J, Lees KR, Tyrrell PJ, et al. Detection of atrial fibrillation after ischemic stroke or transient ischemic attack: A systematic review and meta-analysis. Stroke 2014; 45:520-6.

6. Sanna T, Diener HC, Passman RS, DiLazzaro V, Bernstein RA, Morillo $\mathrm{CA}$, et al. Cryptogenic stroke and underlying atrial fibrillation. N Engl J Med 2014; 370:2478-86.

7. Sinner MF, Tucker NR, Lunetta KL, Ozaki K, Smith JG, Trompet S, et al. Integrating genetic, transcriptional, and functional analyses to identify 5 novel genes for atrial fibrillation. Circulation 2014; 130:1225-35.

8. Tada H, Shiffman D, Smith JG, Sjogren M, Lubitz SA, Ellinor PT, et al. Twelve-single nucleotide polymorphism genetic risk score identifies individuals at increased risk for future atrial fibrillation and stroke. Stroke 2014; 45:2856-62.

9. Wang J, Bai Y, Li N, Ye W, Zhang M, Greene SB, et al. Pitx2micro RNA pathway that delimits sinoatrial node development and inhibits predisposition to atrial fibrillation. Proc Natl Acad Sci USA 2014; 111:9181-6.

10. Olesen MS, Nielsen MW, Haunso S, Svendsen JH. Atrial fibrillation: The role of common and rare genetic variants. Eur J Hum Genet 2014; 22:297-306.

11. Parvez B, Shoemaker MB, Muhammad R, Richardson R, Jiang L, Blair MA, et al. Common genetic polymorphism at $4 q 25$ locus predicts atrial fibrillation recurrence after successful cardioversion. Heart Rhythm 2013; 10:849-55.

12. Benjamin Shoemaker M, Muhammad R, Parvez B, White BW, Streur M, Song Y, et al. Common atrial fibrillation risk alleles at $4 q 25$ predict recurrence after catheter-based atrial fibrillation ablation. Heart Rhythm 2013; 10:394-400.

13. Parvez B, Vaglio J, Rowan S, Muhammad R, Kucera G, Stubblefield $\mathrm{T}$, et al. Symptomatic response to antiarrhythmic drug therapy is modulated by a common single nucleotide polymorphism in atrial fibrillation. J Am Coll Cardiol 2012; 60: 539-45.

14. Fromm MF. Genetically determined differences in P-glycoprotein function: Implications for disease risk. Toxicology 2002; 181-182:299-303.

15. Eichelbaum M, Fromm MF, Schwab M. Clinical aspects of the MDR 1 (ABCB1) gene polymorphism. Ther Drug Monit 2004; 26:180-5.

16. Greiner B, Eichelbaum M, Fritz P, Kreichgauer HP, von Richter $O$, Zundler $\mathrm{J}$, et al. The role of intestinal P-glycoprotein in the interaction of digoxin and rifampin. J Clin Invest 1999; 104: 147-53.

17. Hoffmeyer S, Burk O, von Richter O, Arnold HP, Brockmöller J, Johne A, et al. Functional polymorphisms of the human multi drug-resistance gene: Multiple sequence variations and correlation of one allele with pglycoprotein expression and activity in vivo. Proc Natl Acad Sci USA 2000; 97:3473-8.

18. Kurata $\mathrm{Y}$, leiri I, Kimura M, Morita T, Irie S, Urae A, et al. Role of human MDR 1 gene polymorphism in bioavailability and interaction of digoxin, a substrate of p-glycoprotein. Clin Pharm Ther 2002; 72:209-21.

19. leiri I, Takane H, Ofsuba K. The MDR 1 (ABC1) gene polymorphism and its clinical implications. Clin Pharmacokinet 2004; 43:553-76. 